\title{
CONSCIÊNCIA INTEROCETIVA E FUNCIONAMENTO SEXUAL FEMININO: REVISÃO SISTEMÁTICA
}

\author{
Celina Ribeiro \\ ISPA - Instituto Universitário, Rua Jardim do Tabaco \\ Lisboa - Portugal \\ celine_19r@hotmail.com \\ Henrique Pereira \\ Departamento de Psicologia e Educação da Faculdade de Ciências Sociais e Humanas da Universidade da Beira \\ Interior \\ Covilhã - Portugal \\ CIDESD - Centro de Investigação em Desporto, Saúde e Desenvolvimento Humano, Vila Real - Portugal \\ hpereira@ubi.pt
}

Recepción Artículo: 10 mayo 2021 Admisión Evaluación: 10 mayo 2021 Informe Evaluador 1: 14 mayo 2021 Informe Evaluador 2: 22 mayo 2021 Aprobación Publicación: 01 junio 2021

\section{RESUMO}

A atenção prestada aos sinais internos do corpo parece ter uma influência importante no funcionamento sexual da mulher, uma maior consciência interocetiva parece estar relacionada com um melhor funcionamento sexual, nas suas diversas dimensões. Neste sentido, pretendeu-se identificar, selecionar, avaliar e sintetizar as evidências científicas relevantes disponíveis sobre a relação da consciência interocetiva e o funcionamento sexual nas mulheres, através de uma revisão sistemática da literatura. Para a revisão foi levantada a pergunta de investigação: A consciência interocetiva apresenta uma influência relevante no funcionamento sexual feminino? Os estudos foram considerados elegíveis se se referissem à relação da consciência interocetiva ou mindfulness com aspetos do funcionamento sexual feminino, se fossem estudos empíricos, com uma amostra não-clínica. Foi realizada a pesquisa científica em várias bases de dados, a partir de palavras-chave e conceitos-chave. Foram selecionados sete artigos cientííicos, que foram analisados e sintetizados. Todos os artigos são concordantes na importância da consciência interocetiva nos vários aspectos do funcionamento sexual avaliados, afetando também fatores psicológicos como a ansiedade, limitações psicológicas e satisfação sexual. Aumentar a consciência interocetiva pode aumentar o bem-estar sexual de mulheres que não fazem parte de uma população clínica, podendo mesmo protegê-las de disfunções sexuais futuras.

Palavras-chave: consciência interocetiva; funcionamento sexual; mindfulness 


\title{
CONSCIÊNCIA INTEROCETIVA E FUNCIONAMENTO SEXUAL FEMININO: REVISÃO SISTEMÁTICA
}

\begin{abstract}
Interoceptive awareness and female sexual functioning: Systematic review. The attention paid to internal signs seems to have an important influence on the sexual functioning of women, a greater interocective awareness seems to be related to better sexual functioning, in its various dimensions. In this sense, it was intended to identify, select, evaluate and synthesize the relevant scientific evidence available on the relationship of interocective awareness and sexual functioning in women, through a systematic review of the literature. For the review, the following research question was raised: Does interocective awareness have a relevant influence on female sexual functioning? Studies were considered eligible if they referred to the relationship between interoceptive awareness or mindfulness with aspects of female sexual functioning, if they were empirical studies, with a non-clinical sample. Scientific research was carried out in several databases, starting from keywords and certain concepts. Seven scientific articles were selected, which were analyzed and synthesized. All articles are in agreement on the importance of interocective awareness in the various aspects of sexual functioning evaluated, also affecting psychological factors such as anxiety, psychological limitations, sexual satisfaction. Increasing interocentive awareness can increase the sexual well-being of women who are not part of a clinical population, and may even protect them from future sexual dysfunctions.
\end{abstract}

Keywords: interoceptive awareness; sexual functioning; mindfulness

\section{INTRODUÇÃO}

A sexualidade faz parte da intimidade emocional e física, estando presente na vida de qualquer pessoa, independentemente da forma como é vivida ou experienciada. A sexualidade é, ainda, constituída por diversas dimensões que a tornam complexa e subjetiva, sendo influenciada por vários fatores, sejam eles biológicos, psicológicos ou sociais. A interoceção é o processamento e a representação dos sinais corporais internos aferentes e quando as pessoas se referem às suas emoções, estão também a recorrer e a perceber as sensações corporais internas, necessárias para descrever as suas emoções (Nummenmaa, Glerean, Hari \& Hietanen, 2014). Esta associação entre a interoceção e as emoções pode explicar a forma como as respostas interocetivas são percebidas individual e idiossincráticamente, pois as emoções são construções individuais do mundo. Contudo, é importante perceber que as respostas interocetivas podem ser semelhantes, mas serem categorizadas emocionalmente de maneiras diferentes (Barrett, 2017). Neste sentido, torna-se relevante conjugar as mudanças fisiológicas à experiência emocional, de forma a dar base às propostas teóricas que defendem a existência de uma base interocetiva nas respostas emocionais (Critchley \& Garfinkel, 2017).

As emoções são fatores cruciais na medida em que podem influenciar o comportamento sexual diretamente, aumentando ou diminuindo os sentimentos de excitação sexual e desejo sexual, pois a consciencia das emocoes, bem como as sensacoes corporais internas, são um fator determinante no bom funcionamento sexual (Graham, 2010). Ainda assim, o conceito de "interoceção" é relativamente recente na literatura académica, sendo que o seu significado e definição operacional ainda são consensuais (Ceunen, Vlaeyen \& Diest, 2016). 0 que é geralmente aceite pela maioria dos investigadores atuais, é que a interoceção é a perceção do estado do corpo, ou seja, a perceção do que acontece no interior do nosso organismo (quando percebemos fome, sede, quando nos damos conta que respiramos, dos nossos batimento cardíaco, quando sentimos excitação, fadiga, quando damos conta de uma determinada temperatura, dor, etc.) (Craig, 2002). A par do conceito de interoceção, é imprescindível referir 0 conceito de mindfulness, sendo que este último se remete à consciência corporal, nomeadamente à atencao ou perceção da experiência interna das sensações orgânicas, tendo um papel relevante na consciência corporal ou na perceção dos sinais do corpo, que define a interoceção (Mehling et al., 2009), ou seja, a interoceção é uma componente chave do mindfulness.

As investigações mostram que, no caso das mulheres, quando estas apresentam baixa consciência interocetiva estão mais propensas a ter sintomas clínicos como depressão, baixa auto-estima, ansiedade, julgamento negativo de si mesmo, que impedem o desejo sexual, sendo que o mindfulness parece melhorar estes sintomas (Brotto, 2018). Existem ainda evidências de que, nas mulheres, quando aplicado o mindfulness existem melho- 
rias significativas na ligação mente/corpo, ou seja, existem melhorias na consciência interocetiva (Handy \& Meston, 2016). Além disso, estas melhorias refletem-se em melhorias também no desejo sexual e na angústia sexual relatada (Brotto 2018). De facto, vários estudos com amostras clínicas de mulheres com perturbações sexuais, mostraram que a intervenção com mindfulness apresenta melhorias no funcionamento sexual (Brotto \& Heiman 2006; Brotto, Basson \& Luria, 2008; Brotto, Seal \& Rellini, 2012; Brotto et al., 2012; Brotto. Basson, Smith, Driscoll \& Sadownik, 2015; Paterson, Handy \& Brotto, 2016).

0 grau de concordância entre a excitação sexual autorreferida e a resposta sexual psicofisiológica, pode apresentar relevância no entendimento do funcionamento sexual saudável, nomeadamente as mulheres (Brotto, 2016), ainda que a concordância sexual possa ser um fenómeno distinto da interoceção e, desta forma, necessita da sua própria explicação (Suschinsky \& Lalumière, 2013). Se, por um lado, algumas investigações mostraram que a concordância sexual não tinha associação significativa com consciência corporal (Suschinsky \& Lalumière, 2012; Suschinsky \& Lalumière, 2013; Velten \& Brotto, 2017), por outro, outras mostraram que níveis mais elevados de interoceção estavam ligados a uma associação mais forte entre excitação percebida e fisiológica (por exemplo, fluxo sanguíneo genital)/e genital (Handy \& Meston, 2016); Brotto, Seal \& Rellini, 2012; Brotto, Chivers, Millman \& Albert, 2016). Handy \& Meston, 2016). De qualquer modo, 0 que fica demonstrado é que maiores níveis de interoceção estão ligados a relações mais fortes entre a excitação percebida e a fisiológica, mas não entre a excitação subjetiva e genital (Handy \& Meston, 2018). Dada a escassez de informação científica sobre esta temática, desenvolveu-se o presente trabalho cujos objetivos se apresentam a seguir.

\section{OBJETIVOS DA INVESTIGAÇÃO}

0 principal objetivo deste estudo é realizar uma revisão sistemática de estudos científicos publicados até ao momento, acerca da relação que possa existir entre a consciência interocetiva e aspetos do funcionamento sexual feminino. Estes dados permitirão desenvolver estratégias/recomendações ou planos de intervenção terapêutica mais eficazes, baseados na consciência interocetiva para melhorar o funcionamento sexual nas mulheres, apresentando consequências positivas paras as suas emoções e melhorias na sua qualidade de vida sexual e qualidade de vida em termos gerais.

\section{AMOSTRA}

Nesta revisão sistemática foram selecionados 7 artigos científicos ( $n=7)$, que abordavam estudos empíricos sobre a relação da consciência interocetiva (ou mindfulness) no funcionamento sexual de mulheres, com amostras não-clínicas.

\section{METODOLOGIA}

Partindo-se da análise dos pressupostos teóricos, definiram-se as questões a serem respondidas: A consciência interocetiva apresenta uma influência relevante no funcionamento sexual feminino? Que tipo de impacto tem a consciência interocetiva no funcionamento sexual feminino? A pesquisa foi efetuada em diversas bases de dados (APA Psyclnfo, APA PsycArticles, Regional Business News, APA PsycBooks, Psychology and Behavioral Sciences Collection, PEP Archive, MEDLINE, Academic Search Complete, Business Source Complete, Library, Information Science \& Technology Abstracts, eBook Collection (EBSCOhost), ERIC) com palavras-chave e conceitos previamente definidos: "interoceptive awareness" OR "body awareness", AND "sexual" (resultaram 413 artigos) e "mindfulness" AND "sexual" (resultaram 219). Verificou-se que ainda não foram elaboradas revisões sistemáticas especificamente sobre o tema em estudo. De modo a obter uma maior objetividade e validade do processo de revisão, foi primeiramente desenvolvido um protocolo, de forma a definir os objetivos, perguntas de investigação, critérios de elegibilidade, estratégia de pesquisa e análise da qualidade dos estudos incluídos e da própria revisão. Quanto aos critérios de elegibilidade, foram tidos em conta os seguintes critérios de inclusão: trabalhos que analisavam a consciência interocetiva no funcionamento sexual feminino (foram incluídos estudos que abrangiam o funcionamento sexual masculino, desde que houvesse uma discriminação em relação ao fun- 


\section{CONSCIÊNCIA INTEROCETIVA E FUNCIONAMENTO SEXUAL FEMININO: REVISÃO SISTEMÁTICA}

cionamento sexual feminino, analisando este último de forma individual), incluíam amostras não-clínicas, escritos em português, inglês, espanhol ou francês. Não houve limitação de nacionalidade na consideração dos participantes dos estudos. Consideraram-se como critérios de exclusão os seguintes: não serem estudos empíricos e terem amostras apenas clínicas. Não foram colocadas restrição quanto à data.

\section{RESULTADOS}

Os 413 mais 219 estudos obtidos foram analisados individualmente com base nos seus títulos e resumos. Os estudos potenciais foram posteriormente submetidos a uma leitura do texto integral. Foram selecionados 7 artigos que cumpriam integralmente os critérios de inclusão, a partir dos quais foi extraída a informação dos artigos de forma sintética e avaliada a qualidade das evidências. A extração dos dados foi realizada com base nos objetivos do estudo, métodos de estudo, amostra, instrumento, resultados de interesse e principais conclusões.

Dos sete estudos, verificou-se que três deles apresentavam como objetivo analisar a relação existente entre a consciência interocetiva e o funcionamento sexual, sendo que um deles abordou a concordância sexual. Os outros três estudos pretendiam analisar o impacto do treino de atenção plena (mindfulness) no funcionamento sexual, dois deles abordavam a questão da concordância sexual, elevando a três estudos, no total, que abordavam esta questão. Um dos estudos pretendeu analisar o treino de atenção plena (mindfulness) na capacidade de as mulheres perceberem a sua própria resposta corporal a estímulos sexuais (consciência interocetiva a nível sexual).

Todos os estudos abordavam amostras de mulheres funcionais (ainda que pudessem incluir amostras clínicas, ou com homens, que não seriam consideradas nos resultados de interesse). Em todos os estudos a idade mínima foi de 18 anos e a idade máxima foi de 72 anos. No total, participaram 1174 mulheres, somando as participantes de todos os setes estudos. Quanto aos instrumentos, todos os estudos aplicaram instrumentos de autorrelato e quatro deles recorreram a medidas objetivos para sinais fisiológicos, conforme se desenvolve a seguir.

Berengue, Rebôlo e Costa (2019), desenvolveram um estudo com o objetivo de analisar as relações da perceção interocetiva e da alexitimía com várias dimensões do funcionamento sexual, recorrendo a uma amostra não clínica de ambos os sexos. A amostra incluiu 228 mulheres portuguesas (a partir dos 18 anos, $M=24.6$ ). Os instrumentos utilizados foram o Toronto Alexithymia Scale (TAS-20), Multidimensional Assessment of Interoceptive Awareness (MAIA), Female Sexual Function Index (FSFI) e o Female Sexual Distress Scale-Revised (FSDS-R) para as mulheres. Quanto aos resultados, verificou-se maior consciência interocetiva correlacionada positiva e significativamente com melhor funcionamento sexual feminino em todas as dimensões: menor angústia sexual, maior desejo, maior excitação, melhor lubrificação, maior capacidade de orgasmo, maior satisfação e menos dor.

Carvalheira, Price e Neves (2017), realizaram um estudo, cujo objetivo foi explorar as potenciais diferenças de consciência corporal e dissociação corporal entre homens e mulheres com e sem dificuldades sexuais.A amostra foi constituída por 909 participantes (445 eram mulheres portuguesas, com idade entre os 18 e 72 anos, ( $M=34.08)$. Os instrumentos utilizados foram o Body Awareness and Bodily Dissociation, Distressing Sexual Difficulties, Sexual Trauma, Sexual Activity in the Previous 12 Months. As mulheres sem dificuldades sexuais tiveram consciência corporal significativamente maior em comparação com as mulheres com dificuldades. Os resultados destacaram a relação entre dissociação corporal (antítese de consciência interocetiva) e dificuldades sexuais entre as mulheres. As mulheres que relataram falta de interesse sexual ou de excitação sexual tiveram níveis significativamente mais baixos de consciência corporal e níveis significativamente mais altos de dissociação corporal, em comparação com mulheres que não relataram essas dificuldades sexuais. As mulheres que relataram ansiedade durante a relação sexual e dificuldade para atingir o orgasmo apresentaram níveis significativamente mais elevados de dissociação corporal em comparação com as mulheres que não relataram essas dificuldades. 
Silverstein, Brown, Roth e Britton (2011), investigaram o efeito do treino de meditação mindfulness na capacidade das mulheres de registar a sua própria resposta corporal a estímulos sexuais, ou consciência interocetiva. A amostra foi composta por 44 estudantes universitários (dos quais 30 mulheres, idades entre os 18 e 22 anos), que participaram num curso de 12 semanas de meditação ou de um curso de controlo ativo, com conteúdo ou formato de laboratório semelhantes. A consciência interocetiva foi medida pelo tempo de reação na avaliação da resposta fisiológica aos estímulos sexuais. As limitações psicológicas foram avaliadas com medidas autorrelatadas de atenção plena e bem-estar psicológico. Foram utilizadas as escalas: Mindful Attention Awareness Scale (MAAS), Five Facet Mindfulness Questionnaire (FFMQ), Scales of Psychological Well-being, Brief Symptom Inventory (BSI) e medidas objetivas. Foi apresentado, aos participantes, uma série de fotos selecionadas do International Affective Picture System, usando o software DMDX (60). Os participantes foram instruídos a avaliar a intensidade de sua resposta fisiológica a slides agradáveis, desagradáveis e neutros, através de uma escala de excitação. As mulheres que participaram no treino de meditação, tornaram-se significativamente mais rápidas no registo das suas respostas fisiológicas (consciência interocetiva) aos estímulos sexuais em comparação com 0 grupo de controlo. Além disso, verificou-se que as melhorias na consciência interocetiva foram correlacionadas com melhorias nas limitações psicológicas (atenção, autojulgamento e sintomas clínicos) em relação ao funcionamento sexual saudável. As mulheres que meditaram melhoraram a sua habilidade de perceber e diferenciar sensações corporais (interoceção) e aumentaram a sua capacidade de vivenciar os seus estados internos com menos julgamento e mais auto-aceitação.

Handy e Meston (2016), desenvolveram um estudo com o objetivo de perceber até que ponto as mulheres conseguiam perceber as suas sensações de excitação genital, o papel que a consciência interocetiva desempenhava nessa perceção e se a consciência interocetiva influenciava a concordância sexual (associação entre excitação sexual subjetiva e fisiológica). Participaram 26 mulheres sexualmente funcionais (com idade entre os 1821 anos, $M=19$ ). As participantes assistiram a um filme erótico enquanto os seus níveis fisiológicos e percebidos de excitação sexual genital eram medidos continuamente. Recorreu-se a um fotopletismógrafo vaginal, um "arousometer" (rato de computador conectado a uma alavanca numerada de 0 (sem excitação genital) a 7 (excitação genital máxima), que o participante move durante a apresentação dos estímulos para indicar mudanças na excitação sexual), recorreu-se a uma escala cinematográfica de Heiman e Rowland, a um questionário de atenção às pistas genitais, ao MAIA e ao FSFI. Os resultados indicaram uma relação linear entre a excitação genital percebida e fisiológica, bem como se verificou que a consciência interocetiva influenciava essa relação. As mulheres com maior perceção global do corpo exibiram uma relação mais forte entre a excitação genital fisiológica e subjetiva/percebida. No entanto, examinando a relação entre excitação fisiológica e subjetiva, usando análises correlacionais, não houve correlação significativa (Handy \& Meston, 2016).

No estudo de Velten, Brotto, Chivers, Hirschfeld e Margraf (2020), o objetivo foi investigar o impacto de três exercícios de atenção plena na resposta sexual das mulheres, nomeadamente na excitação sexual genital feminina, na excitação sexual subjetiva e concordância sexual. Participaram 49 mulheres (amostra não-clínica, com idade entre os 18-41 anos, $M=25.22$ ). Os instrumentos utilizados foram um "arousometer", que consistia num rato de computador para assinalar alteração na excitação sexual subjetiva, durante a apresentação de filmes eróticos, amplitude do pulso vaginal (VPA), medidas numa escala do tipo Likert, para avaliar quão difícil ( $1=$ extremamente difícil; 9 = nada difícil), relaxante ( 1 = nada relaxante; 9 = extremamente relaxante) e agradável ( $1=$ extremamente desagradável; 9 = extremamente agradável), acharam os exercícios (baseados na atenção plena e numa tarefa de imagens mentais), e responderam à Escala de Mindfulness de Toronto. 0 foco nos órgãos genitais levou a uma maior excitação genital durante o exercício. Já o foco nas sensações do corpo e nos órgãos genitais foi associado a uma maior excitação sexual subjetiva durante os filmes eróticos, ainda que com efeito reduzido. Concluiu-se que o foco da atenção durante uma prática de atenção plena pode afetar diferentemente a excitação sexual genital e subjetiva. Contudo, a concordância da excitação sexual aumentou após os exercícios body-scan (as mulheres foram encorajadas na perceção do seu corpo todo) e leave-on-a-stream (observação do seu fluxo 


\section{CONSCIÊNCIA INTEROCETIVA E FUNCIONAMENTO SEXUAL FEMININO: REVISÃO SISTEMÁTICA}

de pensamentos), mas não após o exercício de sensações sexuais (focalizar a atenção nos órgãos genitais como um todo e em sensações nas áreas específicas de seus órgãos genitais).

Velten, Margraf, Chivers e Brotto (2018), levaram a cabo um estudo cujo principal objetivo foi investigar 0 impacto de uma tarefa de atenção plena (em laboratório) versus um exercício de visualização, na sexualidade subjetiva e genital, bem como a concordância da excitação sexual em 41 mulheres (amostra não-clínica, com idade entre os 19-43 anos, $M=27.22)$. Um segundo objetivo era explorar a relação entre as diferentes facetas da atenção plena com a resposta sexual. Os instrumentos utilizados foram um "arousometer", consistia num rato de computador para assinalar alteração na excitação sexual subjetiva, durante a apresentação de filmes eróticos, amplitude do pulso vaginal (VPA) e o Five Facet Mindfulness Questionnaire (FFMQ). Foram realizadas duas sessões de laboratório, cada uma incluindo dois filmes eróticos e uma tarefa de atenção. A excitação sexual subjetiva e genital foi medida continuamente durante a apresentação do estímulo. A tarefa de atenção plena levou a uma maior excitação genital subjetiva, em comparação com a tarefa de visualização, ou seja, a observação sem julgamentos, o foco no momento presente e a aceitação de sensações físicas no corpo, incluindo os órgãos genitais, podem ser eficazes para aumentar a percepção da mulher sobre a excitação sexual. Contudo, a tarefa de atenção plena foi associada à menor excitação sexual genital em comparação com a tarefa de visualização. Quatro das cinco subescalas do FFMQ (saber, observar, agir com consciência, não julgar e não reativar), foram preditores negativos da excitação sexual genital em mulheres. Essas descobertas sugerem que dirigir a atenção para as sensações genitais pode induzir um autofoco que pode inibir, em vez de aumentar, a resposta sexual genital nas mulheres. A concordância da excitação sexual genital e subjetiva (ou seja, concordância) foi maior na condição de atenção plena.

Newcombe e Weaver (2016) exploraram a relação entre atenção plena, distração cognitiva e satisfação sexual. A amostra incluiu 355 mulheres adultas que estavam num relacionamento (com idade entre os 18-59 anos, M=25.20). Os instrumentos utilizados foram o The Mindful Attention Awareness Scale, The Five Facet Mindfulness Questionnaire - Short Form, The Cognitive Distraction Scale e o The New Sexual Satisfaction Scale. Foi avaliada a distração cognitiva durante a atividade sexual, como um mediador potencial. As mulheres que relataram níveis mais elevados de plena consciência relataram níveis mais elevados de satisfação sexual (tanto em termos de experiências e sensações sexuais pessoais, como em reações sexuais do parceiro e frequência / variedade de experiências sexuais), e menos distração cognitiva (devido a preocupações com a aparência, questões de desempenho e distrações do dia a dia) durante a atividade sexual. Mulheres que relataram níveis mais baixos de distração cognitiva durante a interação sexual relataram níveis mais elevados de satisfação sexual. A distração cognitiva mediou a associação entre atenção plena e satisfação sexual. Níveis mais altos de atenção plena demonstraram estar associados a níveis mais baixos de distração cognitiva, sendo que a distração cognitiva está associada a múltiplas preocupações sexuais.

A tabela 1 mostra a análise resumida dos sete estudos. 
Tabela 1. Análise resumida dos estudos.

\begin{tabular}{|c|c|c|c|c|c|}
\hline Autores & Título & Objetivo & Amostra & Instrumentos & Resultados \\
\hline $\begin{array}{l}\text { Berengu } \\
\text { e, } \\
\text { Rebôlo e } \\
\text { Costa } \\
(2019)\end{array}$ & $\begin{array}{l}\text { Interoceptiv } \\
e \\
\text { awareness, } \\
\text { alexithymia, } \\
\text { and sexual } \\
\text { function. }\end{array}$ & $\begin{array}{l}\text { Analisar as } \\
\text { relações da } \\
\text { perceção } \\
\text { interocetiva e } \\
\text { da alexitimía } \\
\text { com várias } \\
\text { dimensões do } \\
\text { funcionament } \\
\text { o sexual. }\end{array}$ & $\begin{array}{l}\text { Amostra } \\
\text { não- } \\
\text { clínica, } \\
\text { incluiu } \\
228 \\
\text { mulheres } \\
\text { portuguesa } \\
\text { s (a partir } \\
\text { dos } 18 \\
\text { anos, } M= \\
24.6) \text {. }\end{array}$ & $\begin{array}{l}\text { Toronto } \\
\text { Alexithymia } \\
\text { Scale (TAS- } \\
\text { 20), } \\
\text { Multidimensio } \\
\text { nal Assessment } \\
\text { of } \\
\text { Interoceptive } \\
\text { Awareness } \\
\text { (MAIA), } \\
\text { Female Sexual } \\
\text { Function Index } \\
\text { (FSFI) e o } \\
\text { Female Sexual } \\
\text { Distress } \\
\text { Scale-Revised } \\
\text { (FSDS-R). }\end{array}$ & $\begin{array}{l}\text { Maior } \\
\text { consciência } \\
\text { interocetiva } \\
\text { correlaciona } \\
\text { da com } \\
\text { melhor } \\
\text { funcioname } \\
\text { nto sexual } \\
\text { feminino } \\
\text { em todas as } \\
\text { dimensões. }\end{array}$ \\
\hline $\begin{array}{l}\text { Carvalhe } \\
\text { ira, Price } \\
\text { e Neves } \\
(2017)\end{array}$ & $\begin{array}{l}\text { Body } \\
\text { awareness } \\
\text { and bodily } \\
\text { dissociation } \\
\text { among } \\
\text { those with } \\
\text { and without } \\
\text { sexual } \\
\text { difficulties: } \\
\text { Differentiati } \\
\text { on using the } \\
\text { Scale of } \\
\text { Body } \\
\text { Connection. }\end{array}$ & $\begin{array}{l}\text { Explorar as } \\
\text { potenciais } \\
\text { diferenças de } \\
\text { consciência } \\
\text { corporal e } \\
\text { dissociação } \\
\text { corporal entre } \\
\text { homens e } \\
\text { mulheres } \\
\text { com e sem } \\
\text { dificuldades } \\
\text { sexuais. }\end{array}$ & $\begin{array}{l}909 \\
\text { indivíduos } \\
, 445 \\
\text { mulheres } \\
\text { portuguesa } \\
\text { s (idade } \\
\text { entre os } 18 \\
\text { e } 72 \text { anos, } \\
M= \\
34.08) .\end{array}$ & $\begin{array}{l}\text { Body } \\
\text { Awareness and } \\
\text { Bodily } \\
\text { Dissociation, } \\
\text { Distressing } \\
\text { Sexual } \\
\text { Difficulties, } \\
\text { Sexual } \\
\text { Trauma, } \\
\text { Sexual Activity } \\
\text { in the Previous } \\
12 \text { Months. }\end{array}$ & $\begin{array}{l}\text { Relação } \\
\text { entre } \\
\text { dissociação } \\
\text { corporal e } \\
\text { dificuldades } \\
\text { sexuais } \\
\text { entre as } \\
\text { mulheres. } \\
\text { As } \\
\text { mulheres } \\
\text { que } \\
\text { relataram } \\
\text { ansiedade } \\
\text { durante a } \\
\text { relação } \\
\text { sexual e } \\
\text { dificuldade } \\
\text { para atingir } \\
\text { o orgasmo } \\
\text { apresentara } \\
\text { m níveis } \\
\text { significativa } \\
\text { mente mais } \\
\text { elevados de } \\
\text { dissociação } \\
\text { corporal. }\end{array}$ \\
\hline $\begin{array}{l}\text { Silverstei } \\
\mathrm{n}, \\
\text { Brown, }\end{array}$ & $\begin{array}{l}\text { Effects of } \\
\text { Mindfulness }\end{array}$ & $\begin{array}{l}\text { Investigar o } \\
\text { efeito do } \\
\text { treino de }\end{array}$ & $\begin{array}{l}44 \\
\text { estudantes } \\
\text { universitár }\end{array}$ & $\begin{array}{l}\text { Mindful } \\
\text { Attention } \\
\text { Awareness }\end{array}$ & $\begin{array}{l}\text { Melhorias } \\
\text { na } \\
\text { consciência }\end{array}$ \\
\hline
\end{tabular}




\begin{tabular}{|c|c|c|c|c|c|}
\hline $\begin{array}{l}\text { Roth e } \\
\text { Britton } \\
(2011)\end{array}$ & $\begin{array}{l}\text { Training on } \\
\text { Body } \\
\text { Awareness } \\
\text { to Sexual } \\
\text { Stimuli: } \\
\text { Implications } \\
\text { for Female } \\
\text { Sexual } \\
\text { Dysfunction }\end{array}$ & $\begin{array}{l}\text { meditação } \\
\text { mindfulness } \\
\text { na } \\
\text { capacidade } \\
\text { das mulheres } \\
\text { de registar a } \\
\text { sua própria } \\
\text { resposta } \\
\text { corporal a } \\
\text { estímulos } \\
\text { sexuais, ou } \\
\text { consciência } \\
\text { interocetiva. }\end{array}$ & $\begin{array}{l}\text { ios (dos } \\
\text { quais } 30 \\
\text { mulheres, } \\
\text { idades } \\
\text { entre os } 18 \\
\text { e } 22 \text { anos). }\end{array}$ & $\begin{array}{l}\text { Scale } \\
\text { (MAAS), Five } \\
\text { Facet } \\
\text { Mindfulness } \\
\text { Questionnaire } \\
\text { (FFMQ), } \\
\text { Scales of } \\
\text { Psychological } \\
\text { Well-being, } \\
\text { Brief Symptom } \\
\text { Inventory } \\
\text { (BSI) e } \\
\text { medidas }\end{array}$ & $\begin{array}{l}\text { interocetiva } \\
\text { foram } \\
\text { correlaciona } \\
\text { das com } \\
\text { melhorias } \\
\text { nas } \\
\text { limitações } \\
\text { psicológicas } \\
\text { em relação } \\
\text { ao } \\
\text { funcioname } \\
\text { nto sexual } \\
\text { saudável. }\end{array}$ \\
\hline $\begin{array}{l}\text { Handy e } \\
\text { Meston } \\
(2016)\end{array}$ & $\begin{array}{l}\text { Interoceptiv } \\
\text { e awareness } \\
\text { moderates } \\
\text { the } \\
\text { Relationshi } \\
\text { p between } \\
\text { perceived } \\
\text { and } \\
\text { physiologic } \\
\text { al genital } \\
\text { arousal in } \\
\text { women. }\end{array}$ & $\begin{array}{l}\text { Analisar até } \\
\text { que ponto as } \\
\text { mulheres } \\
\text { conseguiam } \\
\text { perceber as } \\
\text { suas } \\
\text { sensações de } \\
\text { excitação } \\
\text { genital e } \\
\text { verificar a } \\
\text { concordância } \\
\text { sexual. }\end{array}$ & $\begin{array}{l}26 \\
\text { mulheres } \\
\text { sexualmen } \\
\text { te } \\
\text { funcionais } \\
\text { (com } \\
\text { idade entre } \\
\text { os } 18-21 \\
\text { anos, } \\
M=19 \text { ). }\end{array}$ & $\begin{array}{l}\text { Escala } \\
\text { cinematográfic } \\
\text { a de Heiman e } \\
\text { Rowland, um } \\
\text { questionário de } \\
\text { atenção às } \\
\text { pistas genitais, } \\
\text { Toronto } \\
\text { Alexithymia } \\
\text { Scale (TAS- } \\
\text { 20), } \\
\text { Multidimensio } \\
\text { nal Assessment } \\
\text { of } \\
\text { Interoceptive } \\
\text { Awareness } \\
\text { (MAIA). }\end{array}$ & $\begin{array}{l}\text { Mulheres } \\
\text { com maior } \\
\text { perceção } \\
\text { global do } \\
\text { corpo } \\
\text { exibiram } \\
\text { uma relação } \\
\text { mais forte } \\
\text { entre a } \\
\text { excitação } \\
\text { genital } \\
\text { fisiológica e } \\
\text { subjetiva/pe } \\
\text { rcebida. }\end{array}$ \\
\hline $\begin{array}{l}\text { Velten, } \\
\text { Brotto, } \\
\text { Chivers, } \\
\text { Hirschfel } \\
\text { d e } \\
\text { Margraf } \\
(2020)\end{array}$ & $\begin{array}{l}\text { The power } \\
\text { of the } \\
\text { present: } \\
\text { effects of } \\
\text { three } \\
\text { mindfulness } \\
\text { tasks on } \\
\text { women's } \\
\text { sexual } \\
\text { response. }\end{array}$ & $\begin{array}{l}\text { Investigar o } \\
\text { impacto de } \\
\text { três } \\
\text { exercícios de } \\
\text { atenção plena } \\
\text { na resposta } \\
\text { sexual das } \\
\text { mulheres, e } \\
\text { concordância } \\
\text { sexual. }\end{array}$ & $\begin{array}{l}49 \\
\text { mulheres } \\
\text { (amostra } \\
\text { não- } \\
\text { clínica, } \\
\text { com idade } \\
\text { entre os } \\
18-41 \\
\text { anos, } \\
M=25.22 \text { ). }\end{array}$ & $\begin{array}{l}\text { Escala de } \\
\text { Mindfulness } \\
\text { de Toronto e } \\
\text { medidas } \\
\text { objetivas. }\end{array}$ & $\begin{array}{l}\text { O foco da } \\
\text { atenção } \\
\text { durante uma } \\
\text { prática de } \\
\text { atenção } \\
\text { plena pode } \\
\text { afetar } \\
\text { diferenteme } \\
\text { nte a } \\
\text { excitação } \\
\text { sexual } \\
\text { genital e } \\
\text { subjetiva. }\end{array}$ \\
\hline $\begin{array}{l}\text { Velten, } \\
\text { Margraf, } \\
\text { Chivers e }\end{array}$ & $\begin{array}{l}\text { Effects of a } \\
\text { mindfulness } \\
\text { task on } \\
\text { women's }\end{array}$ & $\begin{array}{l}\text { Investigar o } \\
\text { impacto de } \\
\text { uma tarefa de } \\
\text { atenção plena }\end{array}$ & $\begin{array}{l}41 \\
\text { mulheres } \\
\text { (amostra } \\
\text { não- }\end{array}$ & $\begin{array}{l}\text { Five Facet } \\
\text { Mindfulness } \\
\text { Questionnaire } \\
\text { (FFMQ) e }\end{array}$ & $\begin{array}{l}\text { A tarefa de } \\
\text { atenção } \\
\text { plena levou } \\
\text { a uma maior }\end{array}$ \\
\hline
\end{tabular}




\begin{tabular}{|c|c|c|c|c|c|}
\hline $\begin{array}{l}\text { Brotto } \\
\text { (2018) }\end{array}$ & $\begin{array}{l}\text { sexual } \\
\text { response. }\end{array}$ & $\begin{array}{l}\text { na } \\
\text { sexualidade } \\
\text { subjetiva e } \\
\text { genital, bem } \\
\text { como a } \\
\text { concordância. }\end{array}$ & $\begin{array}{l}\text { clínica, } \\
\text { com idade } \\
\text { entre os } \\
19-43 \\
\text { anos, } \\
M=27.22 \text { ). }\end{array}$ & $\begin{array}{l}\text { medidas } \\
\text { objetivas. }\end{array}$ & $\begin{array}{l}\text { excitação } \\
\text { genital } \\
\text { subjetiva, } \\
\text { em } \\
\text { comparação } \\
\text { com a tarefa } \\
\text { de } \\
\text { visualização } \\
\text {, mas a } \\
\text { menor } \\
\text { excitação } \\
\text { sexual } \\
\text { genital. }\end{array}$ \\
\hline $\begin{array}{l}\text { Newcom } \\
\text { be e } \\
\text { Weaver } \\
(2016)\end{array}$ & $\begin{array}{l}\text { Mindfulness } \\
\text {, cognitive } \\
\text { distraction, } \\
\text { and } \\
\text { sexual } \\
\text { well-being } \\
\text { in women. }\end{array}$ & $\begin{array}{l}\text { Explorar a } \\
\text { relação entre } \\
\text { atenção } \\
\text { plena, } \\
\text { distração } \\
\text { cognitiva e } \\
\text { satisfação } \\
\text { sexual. }\end{array}$ & $\begin{array}{l}355 \\
\text { mulheres } \\
\text { adultas } \\
\text { que } \\
\text { estavam } \\
\text { numa } \\
\text { relação } \\
\text { afetiva } \\
\text { (com } \\
\text { idade entre } \\
\text { os } 18-59 \\
\text { anos, } \\
M=25.20 \text { ). }\end{array}$ & $\begin{array}{l}\text { The Mindful } \\
\text { Attention } \\
\text { Awareness } \\
\text { Scale, The } \\
\text { Five Facet } \\
\text { Mindfulness } \\
\text { Questionnaire } \\
\text { - Short Form, } \\
\text { The Cognitive } \\
\text { Distraction } \\
\text { Scale e o The } \\
\text { New Sexual } \\
\text { Satisfaction } \\
\text { Scale. }\end{array}$ & $\begin{array}{l}\text { Mulheres } \\
\text { que } \\
\text { relataram } \\
\text { níveis mais } \\
\text { elevados de } \\
\text { plena } \\
\text { consciência } \\
\text { relataram } \\
\text { níveis mais } \\
\text { elevados de } \\
\text { satisfação } \\
\text { sexual e } \\
\text { menos } \\
\text { distração } \\
\text { cognitiva } \\
\text { durante a } \\
\text { atividade } \\
\text { sexual. }\end{array}$ \\
\hline
\end{tabular}

\section{DISCUSSÃO}

A presente revisão sistemática procurou analisar a relação entre a consciência interocetiva com o funcionamento sexual feminino, bem como caracterizar essa relação. Foram analisados sete estudos científicos, alinhados com as questões de investigação, sendo que a maioria abordava a importância do treino de atenção plena em questões sexuais.

0 estudo de Berengue, Rebôlo e Costa (2019), verificou maior consciência interocetiva correlacionada positiva e significativamente com melhor funcionamento sexual feminino em todas as dimensões, tal como 0 estudo de Carvalheira, Price e Neves (2017) que mostrou que os níveis de dissociação corporal eram maiores nas mulheres que apresentavam falta de interesse sexual ou de excitação sexual (em comparação com mulheres que não relataram essas dificuldades sexuais), bem como nas mulheres que relataram ansiedade durante a relação sexual e dificuldade para atingir o orgasmo. Estes dados revelam a importância de perceber os sinais corporais, em diversas dimensões do funcionamento sexual feminino. Seal e Meston (2007), defendem que, em mulheres com perturbação sexual, ter consciência do próprio corpo, incrementa a resposta sexual, quando estas não sejam distraídas (Seal \& Meston, 2007), corroborando a importância da consciência corporal na resposta sexual, ainda que especificamente em amostra clínica.

Os quatros estudos desta revisão sistemática, que abordam a consciência interocetiva através do treino de atenção plena (mindfulness), foram concordantes na influência positiva deste treino, nos diversos aspetos do fun- 


\section{CONSCIÊNCIA INTEROCETIVA E FUNCIONAMENTO SEXUAL FEMININO: REVISÃO SISTEMÁTICA}

cionamento sexual avaliados. Silverstein, Brown, Roth e Britton (2011), verificaram que melhorias na consciência interocetiva foram correlacionadas com melhorias nas limitações psicológicas (atenção, autojulgamento e sintomas clínicos) em relação ao funcionamento sexual saudável. Velten, Brotto, Chivers, Hirschfeld e Margraf (2020), concluíram que o foco da atenção durante uma prática de atenção plena pode afetar diferentemente a resposta sexual, levando a um aumento da excitação sexual genital e subjetiva. Velten, Margraf, Chivers e Brotto (2018), verificaram que a observação sem julgamentos, o foco no momento presente e a aceitação de sensações físicas no corpo, incluindo os órgãos genitais, podem ser eficazes para aumentar a percepção da mulher sobre a excitação sexual (não a genital). Newcombe e Weaver (2016), concluíram que as mulheres que relataram níveis mais elevados de plena consciência relataram níveis mais elevados de satisfação sexual (tanto em termos de experiências e sensações sexuais pessoais, como em reações sexuais do parceiro e frequência / variedade de experiências sexuais), e menos distração cognitiva (devido a preocupações com a aparência, questões de desempenho e distrações do dia a dia) durante a atividade sexual. Estes dados são corroborados por diversos estudos, com amostras clínicas de mulheres com perturbações sexuais, que também mostraram que a intervenção com mindfulness apresenta melhorias no funcionamento sexual (Brotto \& Heiman 2006; Brotto, Basson \& Luria, 2008; Brotto, Seal \& Rellini, 2012; Brotto et al., 2012; Basson, Carlson \& Zhu, 2013; Brotto, Basson \& Smith, 2015; Paterson, Handy \& Brotto, 2016).

Relativamente à concordância sexual, Handy e Meston (2016), perceberam que a consciência interocetiva facilitou a concordância sexual das mulheres, de tal forma que as mulheres com maiores pontuações na subescala de percepção tiveram maior concordância entre a excitação subjetiva e fisiológica. Velten, Margraf, Chivers e Brotto (2018), também verificaram que a concordância da excitação sexual genital e subjetiva (ou seja, concordância sexual) foi maior na condição de atenção plena. Outros estudos corroboram que níveis mais elevados de interoceção estão ligados a uma associação mais forte entre excitação percebida e fisiológica (por exemplo, fluxo sanguíneo genital)/e genital (por exemplo, Brotto, Seal \& Rellini, 2012; Brotto, Chivers, Millman \& Albert, 2016). Contudo, Velten, Brotto, Chivers, Hirschfeld e Margraf (2020), corroboraram parcialmente esta ideia, pois nem todos os treinos de atenção plena levaram a essa conclusão, e não concluíram que focar especificamente nos genitais, pode levar as mulheres a estarem mais "sintonizadas" com sua resposta de excitação física. Neste sentido, parece que as técnicas focadas em aumentar a interoceção podem melhorar a percepção da excitação genital em mulheres funcionais.

Os resultados dos estudos analisados mostraram que as intervenções de mindfulness, através do acesso mais direto às sensações corporais, podem melhorar a atenção, consequentemente reduzir o julgamento e autocrítica, apresentando um impacto importante no bem-estar emocional e sexual. Silverstein, Brown, Roth e Britton (2011), verificaram que melhorias na consciência interocetiva foram correlacionadas com melhorias nas barreiras psicológicas, nomeadamente mais autoaceitação, influenciando o funcionamento sexual de forma saudável, e Newcombe e Weaver (2016), verificaram que as mulheres que relataram níveis mais baixos de distração cognitiva durante a interação sexual relataram níveis mais elevados de satisfação sexual. Níveis mais altos de atenção plena demonstraram estar associados a níveis mais baixos de distração cognitiva (também associada ao julgamento e autocrítica), sendo que a distração cognitiva está associada a múltiplas preocupações sexuais. Assim, é possível que as intervenções de atenção plena possam ser particularmente úteis para mulheres que tendem a ter pensamentos negativos (mesmo que não apresentem disfunção sexual), durante as interações sexuais, sendo que a atenção plena tem o potencial de aumentar 0 bem-estar das mulheres em geral e podem protegê-las do desenvolvimento de problemas sexuais. Estes resultados vão ao encontro do estudo de Nobre e Pinto-Gouveia (2008), que mostrou que pensamentos de fracasso e desligamento são menos propensos em mulheres sexualmente saudáveis.

A atenção plena pode contribuir para resultados sexuais saudáveis uma vez que estar mais ciente do presente permite uma melhor regulação da emoção e um comportamento mais intencional (Karremans, Schellekens, \& Kappen, 2017). 
Quanto às limitações do estudo, verificou-se que, os vários estudos selecionados analisam a consciência interocetiva na resposta sexual de uma forma bastante heterogénea, o que pode influenciar os resultados ou as suas interpretações. A maioria dos estudos analisa a consciência interocetiva em reduzidas dimensões da resposta sexual. Quatro dos estudos selecionados analisem a consciência interocetiva no funcionamento sexual recorrendo ao mindfulness, abordando o conceito de uma forma diferente e que pode influenciar os resultados. Os intervalos de idade da amostra, na maioria dos estudos, referem-se a idades relativamente jovens, o que também pode interferir nos resultados. Também os instrumentos utilizados foram relativamente heterogéneos, havendo alguns estudos que recorreram a medidas objetivas.

0 presente estudo traz implicações importantes, enfatizando a eficácia que o treino da interoceção pode ter na alteração de pensamentos negativos ou disfuncionais, com consequências na regulação emocional que se traduzem num melhor funcionamento sexual. 0 presente estudo reforça a ideia que programas ou protocolos de intervenção sexual baseados no aumento da consciência interocetiva, podem trazer melhorias significativas no funcionamento sexual de mulheres, independentemente se têm disfunções sexuais ou não, bem como podem protegê-las de perturbações sexuais futuras.

\section{CONCLUSÕES}

0 funcionamento sexual, bem como a satisfação sexual é influenciado por dimensões emocionais, cognitivas e comportamentais. Aumentar a consciência interocentiva pode aumentar o bem-estar sexual de mulheres que não fazem parte de uma população clínica, podendo mesmo protegê-las de disfunções sexuais futuras. As mulheres que não apresentam problemas sexuais podem experimentar preocupações ou pensamentos negativos que afetam 0 seu bem-estar sexual. Assim, as técnicas para aumentar a atenção plena têm o potencial de aumentar 0 bem-estar das mulheres em geral.

0 papel da consciência interocetiva parece ser relevante na análise e até intervenção do funcionamento sexual no caso das mulheres. Contudo sugerem-se mais estudos no caso das mulheres, por exemplo com a aplicação de treino da atenção plena (mindfulness) em amostras não-clínicas.

A percepção do estado do corpo ou perceber e estar atento ao que acontece no interior do nosso organismo parece facilita um funcionamento sexual adequado e pleno.

\section{REFERENCIAS BIBLIOGRÁFICAS}

Barrett, L. F. (2017). The theory of constructed emotion: an active inference account of interoception and categorization. Social Cognitive and Affective Neuroscience, 12, 1-23. doi: http://dx.doi.org/10.1093/ scan/nsw154.

Berenguer,C., Rebôlo, C., \& Costa, R. M. (2019). Interoceptive awareness, alexithymia, and sexual function. Journal of Sex \& Marital Therapy, 45, 729-738. doi: 10.1080/0092623X.2019.1610128

Brotto, L. A. (2016). Mindfulness-based Sex therapy improves genital-subjective arousal concordance in women with sexual desire/arousal difficulties. Archives of Sexual Behavior, 4, 1907-1921. doi: 10.1007/s10508015-0689-8

Brotto, L. A. (2018). Better Sex Through Mindfulness - How Women Can Cultivate Desire. Canada: Greystone Book.

Brotto, L. A., Basson, R., \& Luria, M. (2008). A mindfulness-based group psychoeducational intervention targeting sexual arousal disorder in women. The Journal of Sexual Medicine, 5 (7), 1646-1659. doi: 10.1111/j.1743-6109.2008.00850.x

Brotto, L. A., Basson, R., Smith, K. B., Driscoll, M., \& Sadownik, L. (2015). Mindfulness-based group therapy for women with provoked vestibulodynia. Mindfulness, 6(3), 417-432. doi: 10.1007/s12671-013-0273-z

Brotto, L., Erskine, Y., Carey, M., Ehlen, T., Finlayson, S.,..., Miller, D. (2012). A brief mindfulness-based cognitive behavioral intervention improves sexual functioning versus wait-list control in women treated for gynecologic cancer. Gynecologic Oncolology,, 125(2), 320-335. doi: 10.1016/j.ygyno.2012.01.035 


\section{CONSCIÊNCIA INTEROCETIVA E FUNCIONAMENTO SEXUAL FEMININO: REVISÃO SISTEMÁTICA}

Brotto, L. A., \& Heiman, J. R. (2006). Mindfulness in sex therapy: Applications for women with sexual difficulties following gynecologic cancer. Sexual and Relationship Therapy, 22, 3 - 11. doi: 10.1080/14681990601153298

Brotto, L. A., Seal, B. N., \& Rellini, A. (2012). Pilot study of a brief cognitive behavioralversus mindfulness-based intervention for women with sexual distress and a history of childhood sexual abuse. Journal of Sex and Marital Therapy, 38, 1-27. doi:10.1080/0092623X. 2011.569636

Brotto, L. A., Chivers, M. L., Millman, R. D., \& Albert, A. (2016). Mindfulness-basedsex therapy improves genital-subjective arousal concordance in women with sexual desire/arousal difficulties. Archives of Sexual Behavior, 45, 1907-1921. doi:10.1007/s10508- 015-0689-8

Carvalheira, A., Price, C., \& Neves, C. F. (2017). Body awareness and bodily dissociation among those with and without sexual difficulties: Differentiation using the Scale of Body Connection. Journal of Sex \& Marital Therapy, 43(8), 801-810. doi:10.1080/0092623X.2017.1299823

Ceunen, E., Vlaeyen, J. W. S., \& Diest, I. V. (2016). On the origin of interoception. Frontiers in Psychology, 7, 1 - 17- doi: 10.3389/fpsyg.2016.00743

Craig, A. D. (2002). How do you feel? Interoception: The sense of the physiological condition of the body. Nature Reviews Neuroscience, 3 (8), 655-666. doi: 10.1038/Nrn894

Critchley, H. D. \& Garfinkel, S. N. (2017). Interoception and emotion. Current Opinion in Psychology, 17, 7-14. doi:http://dx.doi.org/10.1016/j.copsyc.2017.04.020

Graham, C. A. (2010). The DSM Diagnostic criteria for female sexual arousal disorder. Archives of Sexual Behavior, 39, 240-255. doi: 10.1007/s10508-009-9535-1

Handy, A. B., \& Meston, C. M. (2016). Interoceptive awareness moderates the relationship between perceived and physiological genital arousal in women. TheJournal of Sexual Medicine, 13, 1907 -1914. doi: 10.1016/j.jsxm.2016.09.018

Handy, A. B., Stanton, A. M. \& Meston, C. M. (2018). Understanding women's subjective sexual arousal within the laboratory: definition, measurement, and manipulation. Sexual Medicine Reviews, 6, 201-216. doi: 10.1016/j.sxmr.2017.11.001.

Mehling, W., Gopisetty, V., Daubenmier, J., Price, C., Hecht, F. \& Stewart, A. (2009). Body awareness: construct and self-report measures. PLoS One, 4(5). doi:10.1371/journal.pone.0005614

Newcombe, B. C., \& Weaver, A. D. (2016). Mindfulness, cognitive distraction, and sexual well-being in women. The Canadian Journal of Human Sexuality 25(2),99-108; doi:10.3138/cjhs.252-A3

Nobre, P. J., \& Pinto-Gouveia, J. (2008). Differences in automatic thoughts presented during sexual activity between sexually functional and dysfunctional males and females. Journal of Cognitive Therapy and Research, 32, 37-49. doi: 10.1007/s10608-007-9165-7

Nummenmaa L, Glerean E, Hari R, Hietanen,J. K. (2014) Bodily maps of emotions. Proceedings of the National Academy of Sciences of América, 111, 646-651. doi: https://doi.org/10.1073/pnas.1321664111

Paterson, L. Q. P., Handy, A. B., \& Brotto, L. A. (2016). A Pilot study of eight-session mindfulness-based cognitive therapy adapted for women's sexual interest/arousal disorder. The Journal of Sex Research, 54(7), 850861.doi:10.1080/00224499.2016.1208800

Seal, B. N, Meston, C. M. (2007). The impact of body awareness on sexual arousal in women with sexual dysfunction. The Journal of Sexual Medicine, 4, 990-1000. doi: https://doi.org/10.1111/j.17436109.2010.02112_1.x

Silverstein, R. G., Brown, H. A., Roth, H. D., \&PhD, Britton, W. B. (2011). Effects of Mindfulness Training on Body Awareness to Sexual Stimuli: Implications for Female Sexual Dysfunction. Psychosomatic Medicine, 73(9), 817-825. doi:10.1097/PSY.0b013e318234e628.

Suschinsky, K. D., \& Lalumière, M. (2012). Is sexual concordance related to awareness of physiological states? Archives of Sexual Behavior. 41, 199-208. doi: 10.1007/s10508-012-9931-9 
Suschinsky, K. D., \& Lalumière, M. L. (2013). The relationship between Sexual concordance and interoception in anxious and nonanxious women. The Journal of Sexual Medicine, 11, 942-955. doi: 10.1111/jsm.12250

Velten, J., \& Brotto, L. A. (2017). Interoception and sexual response in women with low sexual desire. PLoS ONE 12(10). doi: https://doi.org/10.1371/journal.pone.0203094

Velten, j., Brotto, L. A., Chivers, M. L., Hirschfeld, G., \& Margraf, J.(2020). The power of the present: effects of three mindfulness tasks on women's sexual response. Clinical Psychological Science, 8(1), 125-138. doi: $10.1177 / 2167702619861394$

Velten, j., Margraf, Chivers, M. L., J. P, \& Brotto, L. A. (2018). Effects of a mindfulness task on women's sexual response. The Journal of Sex Research, 55(6), 747-757. doi: https://doi.org/10.1080/00224499.2017.1408768 
